\title{
CORRELATION ANALYSIS FOR TROPICAL FOREST ABOVE GROUND BIOMASS AND VERTICAL REFLECTIVITY PROFILE
}

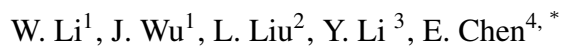 \\ ${ }^{1}$ School of Geographic and Biologic Information, Nanjing University of Posts and Telecommunications, \\ Nanjing, China - liwm@njupt.edu.cn, 1326464135@qq.com \\ ${ }^{2}$ College of Telecommunications and Information Engineering, Nanjing University of Posts and Telecommunications, \\ Nanjing, China - 1219012919@ njupt.edu.cn \\ ${ }^{3}$ Xi’an Zhongke Xingtu Space Data Technology Co. , Ltd., Xi'an, China - 283579773@qq.com \\ ${ }^{4}$ Institute of forest resources information technique, Chinese Academy of Forestry, Beijing, China - chenerx@caf.ac.cn
}

Commission III, WG III/2

KEY WORDS: Tropical forest above ground biomass, SAR Tomography, Capon, vertical reflectivity profile

\begin{abstract}
:
Tropical forests play a key role in the carbon cycle and sustainable development and their above ground biomass (AGB) is applied as an quantitative parameter for the research on global carbon cycle and ecological function. Ten-baseline Polarimetric Synthetic Aperture Radar Interferometry (PolInSAR) images collected in Mondah, Gabon 4th Feb. 2016 during AfriSAR campaign are applied to extract the vertical scattering coefficient with SAR tomography (TomoSAR). Capon is used to reconstruct the vertical reflectivity profile and then the correlation analysis between backscattering coefficients at special height and the in-situ F-AGB is carried out. The results show that Capon performs well when the perpendicular baseline interval is similar and the vertical reflectivity relates closely with the F-AGB in plot scale.
\end{abstract}

\section{INTRODUCTION}

Forests play a key role in the carbon cycle and sustainable development, due to their ability to uptake of carbon dioxide and storage of carbon (Houghton, 2005). As the most abundant and complex forest ecosystem on the earth, tropical forests occupy $40 \%$ of the overall carbon in the world, and their carbon storage is vital for the global carbon cycle and human ecological security. Tropical forest above ground biomass (F-AGB) represents the amount of carbon storage in forests and is therefore applied as an quantitative parameter for the global carbon cycle and ecological function of forest.

Tomographic synthetic aperture radar (TomoSAR) has been widely applied in the 3D reconstruction of complex objects, e.g. urban buildings and forests. It combines the coherent information of the polarimetric SAR interferometry (PolInSAR) or SAR interferometry (InSAR) images in repeat-pass acquisitions to reconstruct reflectivity profiles of volumetric and point-like scatterers in vertical direction (Reigber, Moreira, 2000) (Zhu, Bamler, 2010). Many approaches have been proposed for TomoSAR imaging or vertical reflectivity extraction. Spectral analysis methods such as beamforming (Stoica, Moses, 2005), Capon (Tebaldini, 2010), subspace based method like multiple signal classification (MUSIC) (Frey, Meier, 2011), waveletbased sparse methods such as noise subspace fitting (NSF) and signal subspace fitting (SSF) (Huang et al., 2017), and compressive sensing (CS) approach (Li et al., 2016b), are widely used to extract the distribution of scatterers. The literatures have shown that wavelet-based sparse and CS methods behave well for hard target detection beneath forests, Capon and MUSIC methods do a good job in vertical structure information extraction in forested areas. However, wavelet-based sparse and CS

\footnotetext{
* Corresponding author
}

methods rely on the supposed condition that the reflectivity signal of the distributed media in vertical direction is deterministic and sparse, which restricts their application in forested areas. Meanwhile, Capon and MUSIC methods are subject to the spectral resolution and spectral search.

Tropical forests are dense in vertical direction and their F-AGB may closely relate with the vertical structures. Therefore, to explore the relationship between the tropical F-AGB and vertical structures accurately, reconstruction method with higher vertical resolution is required. In this paper, Capon and MUSIC are applied to extract tropical forest vertical structure. And then the correlation analysis is carried out with the vertical reflectivity profile and the in-situ F-AGB in single pixel and plot scale, respectively.

\section{TEST SITE AND DATA}

\subsection{Test site}

Mondah, Gabon is selected as the test site, and it is a relatively young forest with high variability of density. The test site contains some degraded stands due to the proximity to the city, and the tree height is higher than $40 \mathrm{~m}$ and the mean tree height is about $35 \mathrm{~m}$.

\subsection{Data}

Repeat-pass P-band PolInSAR images collected by the institute of the German Aerospace center (DLR) at 4th February 2016 in Mondah test site during AfriSAR campaign are applied in our study. The flight height is about $6096 \mathrm{~m}$ above average ground level, the P-band operated with a bandwidth of $50 \mathrm{MHz}$ around $435 \mathrm{MHz}$. The PolInSAR images are composed by 9 interferometric slave tracks shifted vertically over the master track, the 
vertical baseline interval is $10 \mathrm{~m}$ and the largest vertical baseline is $80 \mathrm{~m}$. The location of our research is $\mathrm{N}\left(0.52^{\circ}-0.6076\right.$ $\left.{ }^{\circ}\right) \mathrm{E}\left(9.28^{\circ}-9.38^{\circ}\right)$ and the covered area measures approximately $5 \mathrm{Km} \times 8 \mathrm{Km}$ in range and azimuth directions. The master PolInSAR image in pauli-basis is shown in Fig. 1.

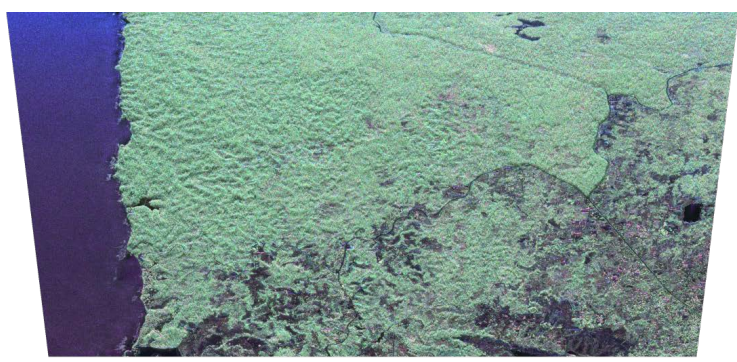

Figure 1. P-band PolInSAR image in Pauli basis in Mondah test site

DEM product with spatial resolution 12.5 m produced by ASF based on ALOS PALSAR is used to assist the transform from slant range to ground range, and part of the image is shown in Fig.2.

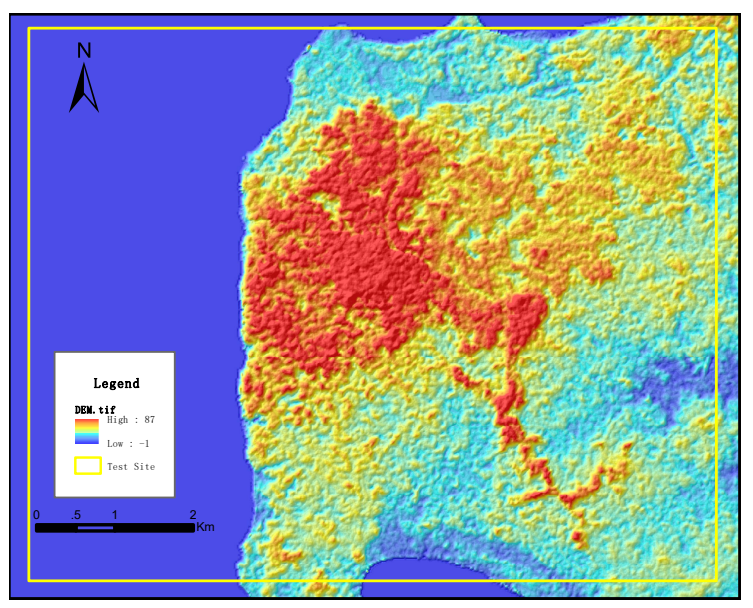

Figure 2. DEM and test site boundary

\section{F-AGB ESTIMATION WITH VERTICAL REFLECTIVITY PROFILE EXTRACTED BY TOMOSAR}

As described in the section 1, tropical forest is rather complex in vertical structures and it is difficult for short wavelength microwave to penetrate through. Therefore, P-band PolInSAR images are applied to complete the task. First, 3D tropical forest is reconstructed with Capon and MUSIC based on repeat-pass P-band PolInSAR images. Second, vertical reflectivity profile is extracted through TomoSAR technique. Finally, correlation analysis is carried out for the in-situ tropical F-AGB and the vertical reflectivity profile in single pixel and plot scale, respectively.

\subsection{Tomographic SAR imaging model}

A stack of $N$ complex PolInSAR images can be obtained by multiple baseline SAR observations over the same objects or areas in repeat-pass mode with slightly different orbit positions. Each perpendicular baseline with respect to the master track is approximate similar, which is represented as $B_{n}$. After fine registration, phase compensation, flat phase removal, and other pre-processing steps, the focused complex value $y_{n}$ of the azimuth-range pixel $\left(x_{0}, r_{0}\right)$ in the $n$th acquisition is

$$
y_{n}=\int_{\triangle z} \gamma(z) e^{\left(-j 2 \pi \xi_{n} z\right)} d z
$$

where $\gamma(z)$ is the reflectivity function along elevations $z$, and $\triangle z$ represents the range of possible elevations. $\xi_{n}=\frac{-2 B_{n}}{\lambda r_{0}}$ is the spatial frequency depending on the elevation aperture position $B_{n}$, range $r_{0}$, and the wavelength $\lambda$. The multi-baseline data acquisition is a randomly sampled Fourier transform of $\gamma(z)$. And the inherent Rayleigh resolution in elevation is represented as $\rho_{z}=\frac{\lambda r}{2 \triangle B}, \triangle B$ is the elevation aperture interval. The interferometric coherence in case of uncorrelated scattering contributions is given as

$$
\mathbf{E}\left[\mathbf{y}_{\mathbf{m}} \mathbf{y}_{\mathbf{n}}^{*}\right]=\int \sigma_{s}^{2}(z) e^{-j 2 \pi \xi_{n} z} d z
$$

where $y_{m}$ is the master image, $y_{n}$ represents the $n$th slave image, and $E[\cdot]$ is the expectation operator, $*$ denotes the conjugation. Eq. 2 shows the phase of interferometric coherence is given by a weighted average of the phases associated with all the scatterers, weights are determined by the backscattered power at each elevation. Eq. 3 is the discrete coherence function through approximately discretizing the continuous interferometric coherence Eq. 2 in elevation $z$ with the unknown noise power $\sigma^{2}$ and identity matrix $I$.

$$
C=\mathbf{E}\left[\mathbf{y}_{\mathbf{m}} \mathbf{y}_{\mathbf{n}}^{*}\right]=\boldsymbol{R} \operatorname{diag}(p) \boldsymbol{R}^{H}+\sigma^{2} I
$$

where $p$ is a nonnegative real vector, $\operatorname{diag}(p)$ is a matrix whose main diagonal equals $p$ and other entries are zeros. The objective of TomoSAR is to estimate the reflectivity profile $\gamma(z)$ in Eq. 1, and $p$ in Eq. 3 for each azimuth-range pixel $\left(x_{0}, r_{0}\right)$ with the covariance matrix of the multi-baseline measured signal $y_{n}$ and $C$ in Eq. 1 and Eq. 3, respectively.

\subsection{Vertical structure extraction}

Vertical structure reconstruction usually achieved by TomoSAR inversion, which can be addressed as spectral estimation problem (Frey, Meier, 2011) (Tebaldini, 2010). The integral in Eq. 2 can be discretized on a set of height $\left\{z_{i}\right\}_{i=1}^{H}$, and obtain the estimation $\operatorname{sigma}_{s}^{2}(z)$ of $\sigma_{s}^{2}(z)$ from the solution of $M^{2}$ equations and $H$ unknowns.

$$
\mathbf{c}=\boldsymbol{A} \boldsymbol{f}+\sigma_{n}
$$

where, $\mathbf{c}=\boldsymbol{v} \boldsymbol{e c}(\boldsymbol{C})$ expresses the vectorization of boldsymbolC, $\boldsymbol{f}$ is a $H$-dimensional column vector with the generic element $[f]_{i}=\sigma_{s}^{2}\left(z_{i}\right) . \sigma_{n}$ represents random noise, and $\boldsymbol{A}$ is the steering matrix defined as

$$
\boldsymbol{A}=\left[e^{j \operatorname{vec}(\boldsymbol{K}) z_{1}}, \ldots, e^{j \operatorname{vec}(\boldsymbol{K}) z_{H}}\right]
$$

where $[\boldsymbol{K}]_{m, n}=k_{z}(m, n)$. In tropical forest, volume scatterers are characterized as continuous vertical distribution of 
backscattered power. To obtain a determined inversion problem for the required $H$, it is required a lot of images focused on the same areas with slightly different positions, which is difficult to meet in practical applications. In practical processes, the number of available images $M$ is too small to tackle this under-determined problem.

There are mainly two approaches to deal with the underdetermination, the first one is to estimate the vertical reflectivity profile as the output power of a filter, and its response is defined by a $M-$ dimensional set of coefficients $h\left(z_{i}\right)$. Therefore, the estimates of $F\left(z_{i}\right)=\sigma_{s}^{2}\left(z_{i}\right)=\mathbf{E}\left\{\left|\boldsymbol{h}^{H}\left(z_{i}\right) y\right|^{2}\right\}$ can be given

$$
\hat{\boldsymbol{F}}\left(z_{i}\right)=h\left(z_{i}\right)^{H} \hat{C} h\left(z_{i}\right)
$$

where the choice of $\boldsymbol{h}(\boldsymbol{z})$ will affect the final vertical resolution, the contrast of $F(z)$ and the estimation performance. Capon is a typical method based on filtering algorithms. The second method is to get the vertical reflectivity profile $\boldsymbol{F}(\boldsymbol{z})$ directly from the Eq. 4 under the assumption that $\boldsymbol{F}(\boldsymbol{z})$ is sparse when expanded onto a given function basis, in which there is only a low number of nonzero needed for the estimation of $F\left(z_{i}\right)$ by using CS techniques.

Using Capon approach, the $\boldsymbol{h}(\boldsymbol{z})$ can be solved by the closedform solution of the minimization problem

$$
\begin{array}{r}
\boldsymbol{h}_{\boldsymbol{C}}(\boldsymbol{z})=\frac{\hat{\boldsymbol{C}}^{-1} \boldsymbol{a}(\boldsymbol{z})}{\boldsymbol{a}^{\boldsymbol{H}}(\boldsymbol{z}) \boldsymbol{C}^{-1} \boldsymbol{a}(\boldsymbol{z})} \\
\text { s.t } \quad \operatorname{argmin}_{h}\left[\boldsymbol{h}^{H}(z) \hat{\boldsymbol{C}} \boldsymbol{h}(\boldsymbol{z})\right] \\
\boldsymbol{h}^{H}(z) \boldsymbol{a}(\boldsymbol{z})=1
\end{array}
$$

where the adaptation filter is guaranteed by the minimization of the true filter output power depending on $\hat{C}$. And then the reflectivity profile $\hat{F_{C}} \hat{(z)}$ can be estimated with Eq. 7 and Eq. 6.

MUSIC is another method to reconstruct the TomoSAR profile with multi-baseline InSAR/PolInSAR data. It is a subspacebased single dimension technique, and its objective function is given by steering vector and the noise space. The formula is given in Eq. 8.

$$
P_{M}(z)=\frac{1}{a \dagger(z) \hat{E_{n}} \hat{E} \dagger_{n} a(z)}
$$

where $\hat{E}_{n}$ is an estimate of the noise subspace. With a least squares estimate the complex reflectivity vector can be obtained as shown in Eq.9.

$$
s(l)=\arg \min _{s}\|y(l)-A(\hat{z}) s\|^{2}
$$

\subsection{The correlations between tropical F-AGB and vertical reflectivity profile}

In our experiment, vertical height interval is set as 0.2 $\mathrm{m}$, and the height range is set as $[-20,60]$, there are 401 vertical reflectivity images extracted from Capon based TomoSAR. To simplify the processing, only the reflectivity at $[-20,-15,-10,5,0,5,10,15,20,25,30,35,40,45,50,60] \mathrm{m}$ height are considered. As the terrain in our test site is rather flat. Vertical reflectivity images in slant range geometry are geocoded onto UTM grids with WGS84 datum by using ALOS PALSAR DEM product and standard GTC lookup tables, which are described in F-SAR system product description (Ponce et al., 2013).

Similar with that in hemi-boreal forest, the tropical F-AGB is estimated from the backscattering powers at special vertical height (Li et al., 2016a), and its formula is shown in Eq. 10.

$$
\begin{array}{r}
\ln (A G B)=a_{0}+a_{1} \times B a_{1}+a_{2} \times B a_{2}+\cdots+a_{n} \times B a_{n} \\
n=[1,2,3]
\end{array}
$$

where $\ln (A G B)$ is the estimated tropical F-AGB expressed in natural logarithm, $a_{n}$ is the coefficient, $B a_{n}$ is the backscattering power at special height correlated closely with F-AGB. In our experiment, 3 images of backscattering power at special height are selected by the backward stepwise regression analysis.

\section{RESULTS AND ANALYSIS}

\subsection{Tropical forest vertical structure information}

The tropical forest vertical reflectivity in $\mathrm{HH} / \mathrm{HV} / \mathrm{VV}$ polarimetric channels along track based on Capon and MUSIC methods are shown in Fig. 3. Fig. 3(a) shows the profile location in master image $\mathrm{HH}$ polarimetric channel, Fig. 3(b) and Fig. 3(c) are the vertical reflectivity profiles in $\mathrm{HH} / \mathrm{HV} / \mathrm{VV}$ polarimetric channels with Capon and MUSIC approach, respectively. It is obviously that the vertical reflectivity profile is continuous for Capon approach, and the vertical reflectivity profile extracted by MUSIC is not continuous, its volume and ground boundaries are obvious.

\subsection{Correlations between F-AGB and vertical reflectivity profiles}

There is four regions of interest (ROI) in-situ tropical F-AGB with $100 \mathrm{~m} \times 100 \mathrm{~m}$ in our test site, and the F-AGB is estimated from ground survey data. There are many subplot $(25 \mathrm{~m} \times 25 \mathrm{~m})$ in each ROI and family, species, stem, locations and so on are surveyed by Fatoyinbo et al. (Fatoyinbo, Jeffery, 2018). Our experiments show that, the vertical backscattering reflectivity has no correlation with the in-situ F-AGB in single tree and pixel scale, this may be caused by the noise or the transformation errors or other factors. The correlation figures in $\mathrm{HH} / \mathrm{HV} / \mathrm{VV}$ channels at $10 \mathrm{~m}$ height are shown in Fig. 4. From the figures, we can see that there is no obvious correlation between the insitu F-AGB and the vertical reflectivity in HH/HV/VV channels at $10 \mathrm{~m}$ height. However, the plot scale vertical backscattering power at height $10 \mathrm{~m}, 25 \mathrm{~m}, 30 \mathrm{~m}$ in $\mathrm{HH} / \mathrm{HV} / \mathrm{VV}$ are obviously related with the in-situ F-AGB in the four ROIs. And the largest correlation coefficient is 0.936 for $\mathrm{VV}$ polarimetric channel, 0.914 for $\mathrm{HV}$ polarimetric channel, and 0.874 for $\mathrm{HH}$ polarimetric channel at $10 \mathrm{~m}$ height. Then the followed correlation coefficients of vertical scattering reflectivity and the insitu F-AGB are $0.848,0.817$ for $\mathrm{VV}$ channel at $25 \mathrm{~m}$ and $30 \mathrm{~m}$ height, and the sixth larger one is 0.802 in $\mathrm{HH}$ channel at $25 \mathrm{~m}$ height. There is an interesting phenomenon that the correction coefficient is higher in VV channel than that in other polarimetric channels for each height profile. By combing the vertical 


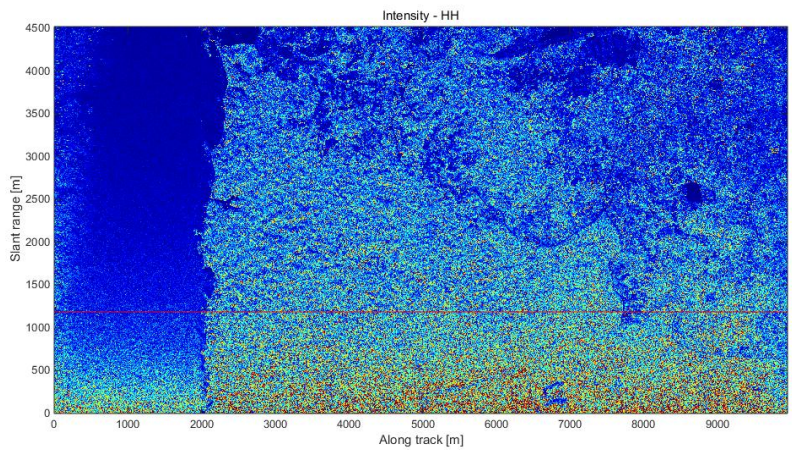

(a)
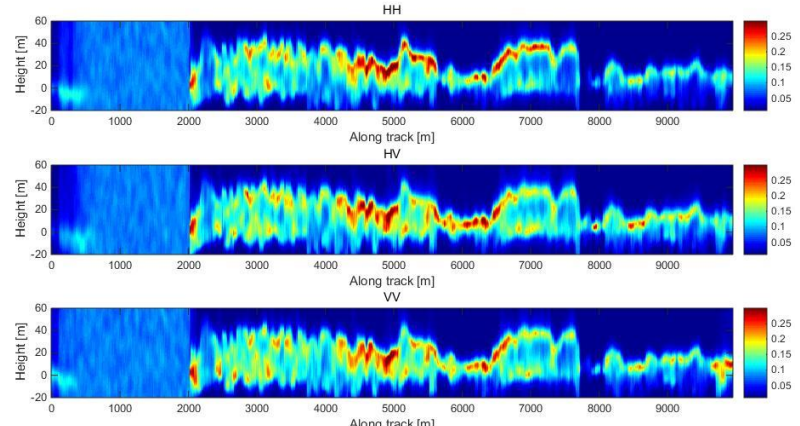

(b)
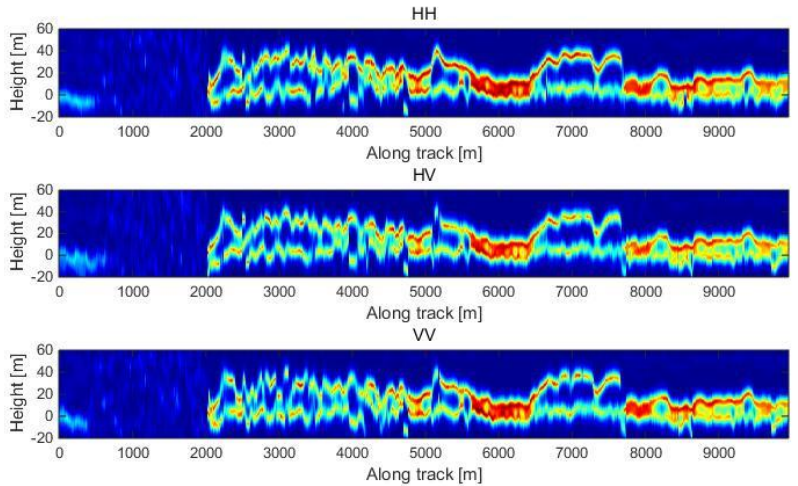

(c)

Figure 3. (a)The profile location in HH polarimetric channel, (b) the vertical reflectivity profiles in $\mathrm{HH} / \mathrm{HV} / \mathrm{VV}$ based on Capon method, and (c)vertical reflectivity profiles in HH/HV/VV based on MUSIC approach. reflectivity coefficients which perform well, the estimated FAGB will get better performance, and its correlation coefficient (R) can reach about to 0.930 .

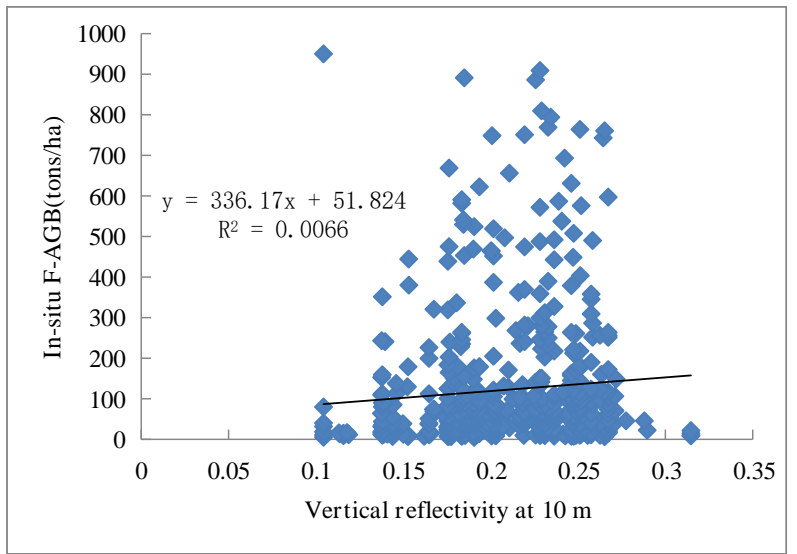

(a)

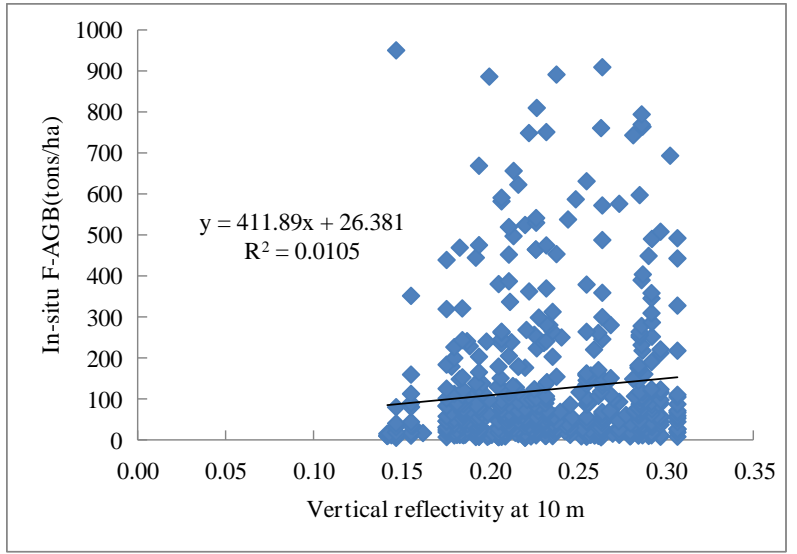

(b)

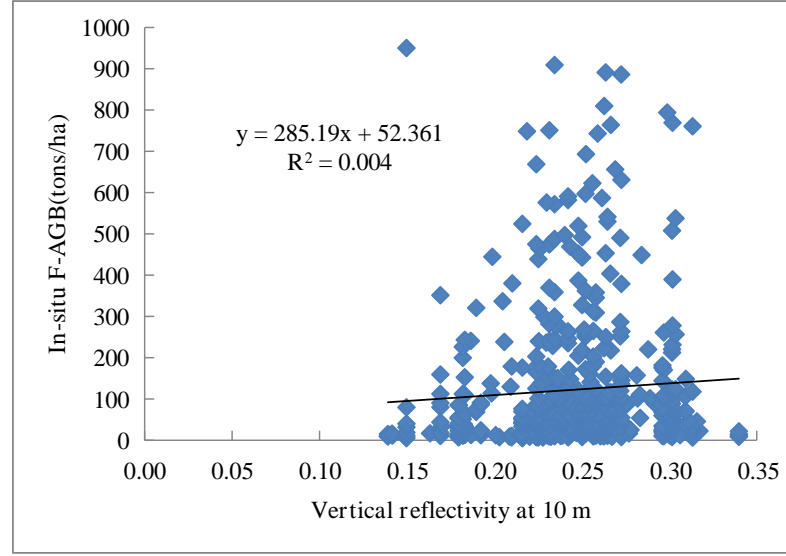

(c)

Figure 4. The correlation coefficient between the in-situ F-AGB and vertical scattering reflectivity at $10 \mathrm{~m}$ height in (a) $\mathrm{HH}$ channel (b) HV channel and (c) VV channel, respectively.

\section{CONCLUSION}

Ten baselines airborne PolInSAR images collected in Mondah, Gabon during 4th Feb. 2016 are applied in our experiment. Capon and MUSIC techniques have been used to reconstruct 
the vertical reflectivity of the forests, and finally the correlations between the tropical in-situ F-AGB and vertical reflectivity profiles are analyzed in single pixel and plot scale, respectively. And the backscattering reflectivity profiles are extracted by Capon in $\mathrm{HH} / \mathrm{HV} / \mathrm{VV}$ channels. The reconstructed vertical reflectivity based on Capon is continuous and little affected by sidelobe, this may indicate that the spatial baselines among all the data are stable, and Capon could obtain good results. The reconstructed vertical reflectivity based on MUSIC is more inclined to locate at ground and volume boundaries, which may indicate this approach is better in ground information extraction and forest height estimation. Tropical F-AGB estimation based on the plot scale backscattering coefficient could obtain a better accuracy, this may indicate that the forest AGB relate closely with the vertical structures in plot scale, and it is similar with that has been described in literatures. What's more, the combined F-AGB estimation model based on several vertical reflectivity with better performance at special height will improve the R to about 0.930 .

However, there are still some problems to be tackled further, such as the in-situ F-AGB has no obvious relations with vertical reflectivity at pixel scale or tree scale at any channels. Meanwhile, although the correlation coefficients between the vertical reflectivity and in-situ F-AGB are higher at special height in plot scale, there are little in-situ data (only 4 ) in plot scale for the model construction. As the reflectivity reconstructed with MUSIC has little information about vertical structure, only the vertical reflectivity extracted by Capon is applied. What's more, the resolution of ALOS PALSAR DEM (12.5 m in spatial) is lower than that of the PolInSAR images (in GTC $2 \mathrm{~m}$ in spatial), which may induce geocoding errors. And it may be the reason for the single pixel scale approach could not be applied into F-AGB estimation.

\section{ACKNOWLEDGEMENTS}

This work was supported by the Project Funded by the Natural Science Foundation of Jiangsu Province under Grant BK20191384, the China Postdoctoral Science Foundation under Grant 2019M661896, and the Nanjing University of Posts and Telecommunications Science Foundation (NUPTSF Grant No. 218085).

\section{REFERENCES}

Fatoyinbo, T., Saatchi, S. S., Armston, J., Poulsen, J., Marselis, S., Pinto, N., White, L. J. T., and Jeffery, K., 2018. AfriSAR: Mondah Forest Tree Species, Biophysical, and Biomass Data, Gabon, 2016. ORNL DAAC, Oak Ridge, Tennessee, USA. https://doi.org/10.3334/ORNLDAAC/1580

Frey, O., Meier, E., 2011. Analyzing tomographic SAR data of a forest with respect to frequency, polarization, and focusing technique. IEEE Transactions on Geoscience and Remote Sensing, 49(10), 3648-3659.

Houghton, R., 2005. Aboveground forest biomass and the global carbon balance. Global Change Biol., 11(6), 945-958.

Huang, Y., Levy-Vehel, J., Ferro-Famil, L., Reigber, A., 2017. Three-dimensional imaging of objects concealed below a forest canopy using SAR tomography at L-band and wavelet-based sparse estimation. IEEE Geoscience and Remote Sensing Let-ter, 14, 1454-1458.
Li, W., Chen, E., Li, Z., Zhang, W., Chang, J., 2016a. Assessing Performance of Tomo-SAR and Backscattering Coefficient for Hemi-Boreal Forest Aboveground Biomass Estimation. Journal of the Indian Society of Remote Sensing, 44(1), 41-48.

Li, X., Liang, L., Guo, H., 2016b. Compressive sensing for multibaseline polarimetric SAR tomography of forested areas. IEEE Transactions on Geoscience and Remote Sensing, 54, 153-166.

Ponce, O., Reigber, A., Prats-Iraola, P., Scheiber, R., Jaeger, M., Pinheiro, M., Horn, R., Keller, M., Fischer, J., 2013. F-sar system - dlr's advanced airborne sar system.

Reigber, A., Moreira, A., 2000. First Demonstration of Airborne SAR Tomography Using Multibaseline L-Band Data. IEEE Transactions on Geoscience and Remote Sensing, 38(5), 2142-2152.

Stoica, P., Moses, R., 2005. Spectral Analysis of Signals. Prince-Hall, Englewook Cliffs, NJ, USA.

Tebaldini, S., 2010. Single and multipolarimetric SAR tomography of forested areas: A parameteric approach. IEEE Transactions on Geoscience and Remote Sensing, 48(5), 2375-2387.

Zhu, X., Bamler, R., 2010. Very High Resolution Spaceborne SAR Tomography in Urban Environment. IEEE Transactions on Geoscience and Remote Sensing, 48(12), 181-184. 